Service social

\title{
L'intervention sociale contre le racisme : sortir du multiculturalisme et de la pensée magique
}

\section{Sylvie Perras et Nicole Boucher}

Volume 42, numéro 1, 1993

Culture et intervention

URI : https://id.erudit.org/iderudit/706602ar

DOI : https://doi.org/10.7202/706602ar

Aller au sommaire du numéro

\section{Éditeur(s)}

École de service social de l'Université Laval

ISSN

1708-1734 (numérique)

Découvrir la revue

Citer cet article

Perras, S. \& Boucher, N. (1993). L'intervention sociale contre le racisme : sortir du multiculturalisme et de la pensée magique. Service social, 42(1), 101-119. https://doi.org/10.7202/706602ar
Résumé de l'article

La lutte contre le racisme est devenu petit à petit l'un des objectifs du service social. Or, selon nous, même si les comportements racistes sont souvent présentés comme un exutoire aux problèmes économiques, le racisme contemporain découle principalement de la logique adoptée par l'État pour faire face aux nouvelles immigrations et à leur intégration dans la société Ainsi, dans un contexte politique modelé par des revendications identitaires multiples, la politique canadienne du multiculturalisme stigmatise encore davantage ceux qu'elle prétend intégrer et de ce fait alimente la résurgence du racisme et complique l'intervention.

Pour expliciter cette thèse, nous retraçons d'abord la genèse du racisme et son évolution dans nos sociétés occidentales modernes. Ensuite, en contextualisant les nouvelles formes d'immigration dans l'espace national canadien, nous démontrons que la politique publique du multiculturalisme a contribué à créer une impasse sur le plan conceptuel, entravant ainsi l'action pour lutter efficacement contre le racisme. Finalement, pour sortir des impasses du multiculturalisme et de la pensée magique, nous proposons aux travailleurs sociaux un cadre alternatif d'intervention sociale.
Ce document est protégé par la loi sur le droit d'auteur. L'utilisation des services d’Érudit (y compris la reproduction) est assujettie à sa politique d'utilisation que vous pouvez consulter en ligne.

https://apropos.erudit.org/fr/usagers/politique-dutilisation/ 


\section{L'intervention sociale contre le racisme: sortir du multiculturalisme et de la pensée magique}

Sylvie PERRAS

Étudiante à la maîtrise en relations internationales Université Laval

Nicole BOUCHER Travailleuse sociale et sociologue Professeure , École de service social

Université Laval

\section{INTRODUCTION}

Un jour un vieux rabbin demanda à ses élèves comment on déterminerait l'heure où finit la nuit et où commence le jour. Est-ce quand on peut distinguer de loin un chien d'un mouton? Non, dit le rabbin. Est-ce quand de loin on peut distinguer un dattier d'un figuier? demanda un autre élève. Non, dit le rabbin. Mais alors, quand est-ce? demandèrent les élèves. C'est quand tu peux regarder le visage de $\mathrm{n}^{\prime}$ importe quel humain et que tu vois ta sœur ou ton frère. Jusque-là, c'est encore la nuit. (Tugendhat, 1985: 58)

Le racisme est à l'ordre du jour. La couverture médiatique des nombreux événements à caractère raciste accapare l'actualité dans divers coins du monde occidental et nous laisse entrevoir une réalité sociale déplaisante et effrayante. Dorénavant, nul ne se sent vraiment totalement à l'abri d'une guerre civile comme celle qui déchire actuellement l'ancienne Yougoslavie. 
Réminiscences d'un passé noir encore trop vivant dans la mémoire collective et craintes de basculer à nouveau dans une folie purificatrice, les raisons de la réapparition du racisme demeurent, dans un tel contexte, quelque peu tabou.

Or, force est de constater que l'évolution des sociétés modernes n'a pas permis d'évacuer les comportements racistes, pas plus que l'idéologie ellemême. Celle-ci, au contraire, a survécu et sa métamorphose discrète s'est opérée dans la foulée des arguments avancés par les mouvements antiracistes sans que ceux-ci prennent conscience de l'arme à double tranchant ainsi créée.

De plus, la dénonciation scientifique des théories biologiques des races humaines n'a pas eu l'écho escompté dans le corps social. On continue à s'affronter dans un espace limité pour des ressources tout autant limitées. Période de récession et de crise économique oblige, l'intolérance grandit et l'altérité visible de l'Autre est de plus en plus prise à partie. L'étranger est souvent le premier à être expulsé ou à tout le moins exclu de la société, puisqu'il incarne l'usurpateur, celui qui dérange, celui qui n'a pas droit de cité.

À une époque où le système international évolue vers la mondialisation par la voie de l'unification économique, étape ultime du capitalisme, les nouvelles immigrations déstabilisent le jeu sur l'échiquier international. Conséquences de l'asymétrie démographique que les économies du Sud sont incapables d'absorber et du déséquilibre entre le Nord et le Sud quant à la répartition des richesses mondiales, les migrations internationales provoquent des déséquilibres structurels supplémentaires, au Nord comme au Sud.

Au Québec, comme dans les sociétés contemporaines des États du Nord, le problème du racisme est dorénavant associé à des préoccupations pour les flux migratoires sud / nord apparemment irréversibles. Dans un contexte social et politique de plus en plus dominé par l'idéologie du relativisme culturel, les nouvelles immigrations, par le pluralisme qu'elles engendrent, contribuent à fragiliser la structure fondamentale de l'État-nation.

Or, selon nous, bien que les comportements racistes soient souvent présentés comme un exutoire aux problèmes économiques, le racisme contemporain découle principalement de la logique adoptée par l'État pour faire face aux nouvelles immigrations et à leur intégration dans la société. Ainsi, dans un contexte politique modelé par des revendications identitaires multiples, la politique canadienne du multiculturalisme stigmatise davantage ceux qu'elle prétend intégrer et, de ce fait, alimente la résurgence du racisme et complique l'intervention.

Dans le présent article, pour expliciter cette thèse, nous retraçons d'abord la genèse du racisme et son évolution dans nos sociétés occidentales modernes. Ensuite, en contextualisant les nouvelles formes d'immigrations dans l'espace national canadien, nous démontrons que la politique publique du multiculturalisme a contribué à créer une impasse sur le plan conceptuel, entravant ainsi l'action pour lutter efficacement contre le racisme. Finalement, 
pour sortir des impasses du multiculturalisme et de la pensée magique, nous proposons aux travailleurs sociaux un cadre d'intervention sociale alternatif.

\section{LE CONCEPT DE RACISME}

L'usage fréquemment inapproprié du terme « racisme » a favorisé la dissolution progressive de la valeur du concept et a de ce fait créé une ambiguïté persistante quant à la véritable nature du racisme. L'incompréhension du phénomène qui en découle rend d'autant plus difficile une lutte ordonnée et efficace. II apparaît d'ores et déjà que le racisme fait référence à un phénomène plus complexe que ne le laissent croire les explications figées des mouvements antiracistes traditionnels (Taguieff, 1991, II). II convient donc, dès le départ, de clarifier le concept autour duquel s'articule notre réflexion.

À l'origine, la théorie de la race élaborée par le comte de Gobineau dans son Essai sur l'inégalité des races humaines (1853-1855) utilise l'anthropologie physique pour expliquer et justifier les rapports de domination, alors que l'esclavagisme est à son apogée et que le colonialisme et l'impérialisme sont des systèmes d'enrichissement économique et de domination politique autorisés. Elle recourt à un système de valeurs et de normes pour expliquer l'évolution historique en fonction d'un déterminisme biologique (Taguieff, 1987: 138).

Poussée au terme de sa logique, la théorie gobinienne des races alliée à une interprétation du "darwinisme social » accréditant la supposée supériorité aryenne donnera lieu dans l'Allemagne nazie à la démence génocidaire bien connue. Cette débâcle honteuse pour l'Occident et l'humanité en général amènera la communauté internationale à nier tout fondement scientifique à la doctrine biologique de l'inégalité naturelle des races. Ce déni d'existence, confirmé dans la Déclaration sur la race et les préjugés raciaux de 1978 de I'UNESCO, laissait croire en la disparition du racisme comme idéologie. Pourtant, il semble bien qu'au contraire, le phénomène ait survécu grâce à sa capacité de métamorphose.

À cause de ces antécédents historiques, le racisme soit demeure assimilé à toute forme de haine ou de mépris, donc incarne l'intolérance même, soit fait référence presque exclusivement à l'extermination de masse par les Nazis. Ces interprétations limitées occultent cependant toute la zone d'ombre dans laquelle le racisme contemporain déploie ses nouveaux visages.

\section{LE NÉO-RACISME}

Dans les années d'après-guerre, la focalisation des arguments antiracistes sur la thèse scientifique du racisme et le consensus apparent ont apaisé les débats idéologiques autour de la question. Ce relatif relâchement de la vigilance 
a empêché l'anticipation de formes idéologiques «inédites» de racisme. Pourtant, dès le milieu des années 70 , le détournement au profit de la nouvelle droite de l'idéologie différentialiste prônée par les antiracistes aurait dû sonner l'alarme. Ce que l'on a pris pour une simple version modifiée du racisme inégalitaire fondé sur un scientisme biologique (donc inoffensif parce que démenti scientifiquement) était plutôt l'ébauche d'un nouveau racisme différentialiste sur des bases culturalistes.

Discrètement, la nouvelle construction idéologique a opéré trois grands déplacements des concepts de base, attitudes et arguments de l'idéologie raciale traditionnelle. Premièrement, le concept de «race» a glissé vers celui $\mathrm{d}^{\prime}$ « ethnie/culture », érigeant ainsi les fondements d'un racisme sur des bases culturalistes. Deuxièmement, le concept d'inégalité a cédé la place à celui de différence, concept apparemment inoffensif qui soutiendra la formation d'une idéologie identitariste. La transposition dans le champ politique de cette idéologie identitariste par le Front national, en France, dès 1983, puis la capacité de mobilisation du Front national auraient dû susciter une prise de conscience quant à la réalité mouvante du racisme contemporain (Taguieff, 1987: 13-14), mais il n'en fut rien. Finalement, on passe d'une idéologie hétérophobe à une autre, hétérophile, qui prône le «droit à la différence » et ainsi, paradoxalement, on justifie l'inégalité.

En conséquence, le remplacement de la notion zoologique de " race» par celle de «culture » permet la diffusion discrète d'un racisme renouvelé, camouflé derrière des mots et des idées nouvelles. Du même coup, une recontextualisation de la problématique et une révision de l'argumentation anti-universaliste propre aux mouvements antiracistes traditionnels deviennent nécessaires.

\section{L'ARGUMENT DIFFÉRENTIALISTE DANS LE DISCOURS NÉO-RACISTE}

Ironiquement, avant d'être récupéré dans le discours néo-raciste, le relativisme culturel a d'abord été élaboré vers la fin du XIXe siècle en réaction au déterminisme biologique et à l'ethnocentrisme porteur des germes de l'idéologie raciste et il a présidé à la naissance de la tradition antiraciste. Celle-ci s'est appuyée sur trois principes: I'autonomie des phénomènes culturels, le déterminisme culturel comme élément dominant des structures mentales et des formes de vie et l'égalité en valeur de toutes les cultures.

Par la suite, toujours dans la perspective offerte par le relativisme culturel, en s'appuyant sur l'idéologisation de la culture, l'antiracisme s'est attaché à stigmatiser toute action porteuse de ségrégation, qu'elle soit raciale ou sociale, naturalisée par des différences considérées comme légitimes. La conséquence de cet accent mis sur une notion abstraite et idéalisée de la 
culture s'est concrétisée par une explosion de revendications identitaires, valorisant, parfois de manière radicale, la différence. La discrimination induite par le passé est invoquée par certains groupes de victimes pour justifier des demandes de reconnaissance et des demandes de réparation pour les torts historiques par des politiques de discrimination positive. L'ethnicisation des relations politiques et sociales devient alors inévitable (Costa-Lascoux, 1991 : 107).

En effet, ce faisant, le discours raciste contemporain a su récupérer le discours du relativisme culturel et du droit à la différence en le détournant de son sens premier. L'affirmation exclusive des différences est utilisée en réponse à la hantise du «métissage culturel ». La différence s'érige en norme de préservation des entités communautaires. L'écart par rapport à cette norme fait surgir, à juste titre, l'angoisse de la dissolution finale des identités collectives dans un universalisme abstrait. Le néo-racisme exploite ainsi à fond les réalités différentielles immédiates (couleur de peau, accent, langue) pour promouvoir sa défense des identités culturelles supposément menacées et, ce faisant, s'apparente à un antiracisme authentique.

Le néo-racisme s'est donc articulé autour de deux axes principaux empruntés aux antiracistes et au mouvement des droits de la personne, soit la défense des identités culturelles et l'éloge de la différence par le moyen du «droit à la différence». L'idéologie de la différence se situe dans un cadre double, soit, premièrement, les formes contemporaines hégémoniques d'individualisme et, deuxièmement, les réactions ethnistes douces (régionalismes) ou violentes (terrorisme-indépendantisme). Le néo-racisme, quant à lui, utilise concurremment l'éloge de la différence (hétérophilie) ou le rejet de la différence (hétérophobie). Ses deux arguments fétiches consistent dans l'existence d'un seuil de tolérance et dans l'inadmissibilité supposée de certaines catégories de migrants, les non-blancs - ceux de l'autre hémisphère - étant le plus souvent visés.

L'idée du seuil cherche à conforter une majorité par l'érection d'une frontière artificielle qui établit la distance entre les deux univers disparates, le sien et celui de l'Autre. La notion de tolérance devient paradoxale en ce qu'elle n'incite plus au respect de la liberté de penser et d'agir d'autrui, mais prétend éviter l'intolérance en supprimant ou en diminuant son objet. La perception quasi biologique de la réaction d'intolérance accorde à cette dernière une crédibilité et une justification naturelles. Ce détour apparemment scientifique par le culturel pour expliquer l'intolérance justifie un retour au racisme original sous sa forme biologique. L'intolérance devient normale, puisque sa victime en est la cause.

La notion de tolérance est paradoxale en ce qu'elle autorise le racisme en l'assimilant à un phénomène inévitable et elle l'interdit en le contenant par une borne inférieure en deçà de laquelle il convient de supporter les immigrés. (De Pudler, 1991 : 162) 
Par ailleurs, I'absolutisation de la différence s'accompagne d'un postulat d'inadmissibilité. La différence reconnue comme inconvertible ne peut qu'entraîner la volonté d'un rejet de l'Autre, son effacement, son anéantissement imaginaire ou effectif. De nouveaux modes de racialisation par la culture ou par l'ethnie procèdent à la mise à distance de I'Autre, tant par le langage hétérophile (éloge de la différence) que par le langage hétérophobe, entraînant de ce fait une corruption idéologique (Taguieff, 1991, II: 41).

Ainsi, par un paradoxal mimétisme des discours, les racistes et les antiracistes se rejoignent. Les débats sont centrés sur le croisement des questions des identités collectives et leur défense, les droits des peuples, le mélange ou les croisements des cultures, sur l'interculturel et le transculturel (Taguieff, 1987: 17). Le racisme que I'on avait cru pour un temps anéanti survit toujours. Aux côtés d'un racisme d'exploitation (esclavagisme, colonialisme) et $d^{\prime}$ 'un racisme d'extermination (nazisme), un racisme mutant a pris forme en substituant la culture à la race et il se révèle d'autant plus efficace qu'il est plus difficile à reconnaître et à cerner.

\section{LES NOUVELLES IMMIGRATIONS}

Le début des années 70 marque une étape charnière dans I'histoire des migrations internationales. La crise de l'énergie et la forte récession qui l'accompagne mettent au jour les failles du système international. L'interruption momentanée de la croissance économique des pays du Nord révèle la vulnérabilité de leurs économies et l'interdépendance inévitable dans une économie-monde qui s'accommode mal des souverainetés nationales des États-nations. Le phénomène migratoire qui à l'origine devait être conjoncturel (en Europe surtout), afin de combler les besoins en main-d'œuvre des pays demandeurs, s'est transformé avec le temps en donnée structurelle des sociétés d'accueil et des relations économiques et politiques internationales (Bolzmann, 1985: 68).

La fermeture des frontières européennes à l'immigration de maind'œuvre a été suivie d'une certaine convergence des politiques de flux chez les États du Nord. Depuis cette époque, tous accordent une garantie de séjour durable aux étrangers possédant des titres de résident régulier, tous tentent d'empêcher l'entrée de travailleurs non qualifiés, tous accordent une attention particulière à la réunification des familles et à l'accueil des réfugiés politiques et, finalement, tous participent à un degré ou à un autre à la fuite des cerveaux (brain-drain) (Weil, 1992). Ces consensus internationaux sont pourtant le reflet de contraintes de valeurs, de contraintes politiques et de contraintes économiques locales. 
Ce changement d'attitude des pays d'émigration opérera un transfert imprévu du contrôle des flux migratoires, qui se traduira par la sédentarisation des travailleurs étrangers, l'augmentation de l'immigration illégale et l'utilisation du système de réfugié comme ultime recours « légal ». Phénomène activé désormais par une dynamique propre, les nouvelles immigrations s'inscrivent dans un contexte complexe: la répartition inéquitable des richesses mondiales entre le Nord et le Sud, la déstructuration des économies des pays en voie de développement (PVD) accentuée par un essor démographique inquiétant, la restructuration des économies du Nord accompagnée de difficultés d'ajustement social interne, la crise de l'État-providence alors que la mondialisation de l'économie se poursuit à un rythme effréné, laissant dans son sillage des communautés politiques et sociales qui n'arrivent plus à s'adapter à tous ces changements. Dès lors, les nouvelles immigrations se démarquent de celles qui les avaient précédées en ce qu'elles se composent de migrants de races et de cultures diversifiées, de milieux sociaux différents et qu'elles modifient durablement le paysage auquel elles viennent se greffer (Verhaeren, 1990).

Dans un tel contexte, nous sommes en droit de supposer avec Jean Leca (1991 : 40) que la difficulté d'intégration des nouvelles immigrations aux sociétés d'accueil est accrue pour des raisons structurelles qui sapent la cohésion sociale nécessaire à cet accueil :

Or, il est plausible que les bases sociales de cette nécessaire propension à la solidarité viennent à s'affaiblir pour des raisons structurelles tenant à la division du travail, la fragmentation des classes, les différenciations culturelles et l'organisation et la représentation politiques. II n'est pas sans intérêt de les détailler car ce n'est que dans ce cadre général que l'on peut prendre la mesure du problème des nouvelles immigrations trop souvent vues comme une déstabilisation externe de sociétés elles-mêmes stables. C'est en réalité presque le contraire: ces immigrations constituent un problème au moment où (et peut-être parce que) les sociétés européennes font face à des crises de régulation qui les privent des ressources nécessaires pour les traiter.

Le problème du racisme dans les sociétés altérées par les nouvelles immigrations se confond avec celui de l'intégration dans des sociétés récemment devenues multiculturelles et multiraciales. Ce sont les paysages urbains des grandes métropoles du monde industrialisé qui se transforment, remettant en question par leur nouveau visage le sens profond de l'identité collective nationale. Les conflits qui surgissent sont des conflits de nationalité et de citoyenneté et s'expriment au travers des décalages de droits sociaux et politiques, des pratiques discriminatoires ou d'exclusion qui se jouent à I'intérieur d'un État national affaibli dans sa centralité même (Gallisot, 1989).

Dans nos États de droit fondés sur I'idéologie républicaine qui érige en paradigme des droits individuels la rationalité et la liberté, la culture et 
I'histoire cèdent leur importance significative dans la définition du lien collectif au profit de l'universalité de certaines qualités humaines. Dans un tel contexte, la nationalité et la citoyenneté se superposent au point de devenir des concepts interchangeables. En tant que concepts de "clôture sociale » alliant les localisations dans les structures sociales, la division du travail et les clivages culturels (linguistiques, religieux), elles permettent la création de sentiments communautaires d'appartenance et définissent par là même l'Autre. Les formes de stratification sociale qui en découlent ne s'appuient pas sur la nature biologique, ni sur des arguments génétiques, mais sur les modes d'accès au territoire, le droit de résidence, voire le droit de cité (Leca, 1992).

Le pluralisme culturel représente un modèle de construction des identités politiques sur des bases sub ou transnationales (ethnie, langue, religion...) et donne une légitimité internationale aux diasporas nationales (Oriol, 1989). La société compartimentée en solidarités ethniques se doit $\mathrm{d}^{\prime}$ 'assurer une distribution égale des richesses nationales à chaque segment culturel. Le processus de modernisation et de capitalisation qui a accompagné la formation de l'État-nation avait éliminé en bonne partie ce pluralisme grâce à une citoyenneté qui en permettait le nivellement. Le pluralisme actuel est postnational en ce qu'il multiplie les communautés politiques et donc les niveaux de citoyenneté au sein d'un même espace national. Ainsi, une série de groupes d'appartenance plus immédiats et plus concrets se forment au détriment d'une société plus solidaire dont la souplesse autorise la viabilité. Pourtant, même dans cette logique, l'État-nation demeure le lieu privilégié de la construction des identités collectives.

\section{LA POLITIQUE CANADIENNE DU MULTICULTURALISME}

Terre d'accueil depuis ses origines, le Canada a été façonné par l'immigration de peuplement. Malgré cela, le racisme d'une société au départ assez homogène, associé au conservatisme des dirigeants politiques, avait permis la mise en place d'une politique discriminatoire de préservation d'un Canada blanc et britannique dans son essence même, situation qui allait se prolonger jusqu'à la fin de la Deuxième Guerre mondiale. L'apparition d'une volonté politique de concertation au niveau international, associée à l'émergence de nouveaux pays dans la fièvre de la décolonisation, devait modifier l'ordre établi. Sur une scène internationale de plus en plus variée et dans un Commonwealth de plus en plus multiracial, la «White Canada Policy» (Hawkins, 1989: 16-25) contrastait dangereusement avec la nouvelle image internationale positive que le Canada tentait d'acquérir.

Dans un climat international favorable aux droits de la personne et aux droits des minorités, la politique canadienne d'immigration s'articulera donc, 
à partir de 1962, autour des principes suivants: la réunification des familles, les préoccupations relatives aux réfugiés, la non-discrimination et la promotion des objectifs démographiques, économiques, sociaux et culturels du Canada. Cette ouverture permettra l'élargissement du bassin de recrutement, diversifiant par le fait même les sources traditionnelles d'immigrants et par conséquent la composition humaine et sociale de la société d'accueil. La reconnaissance de cette métamorphose sociale opérée par les nouvelles immigrations amènera le gouvernement canadien, inspiré par la logique britannique qui associe immigration et lutte contre la discrimination (Crowley, 1992), à instaurer la politique du multiculturalisme en 1971. II nous est ainsi possible de tracer un parallèle entre la décision canadienne d'institutionnaliser le multiculturalisme et les politiques britanniques de lutte contre la discrimination.

Justifié par la volonté de garantir la liberté culturelle, la justice sociale et l'égalité des chances à l'intérieur du système politique existant, le multiculturalisme procédait cependant d'une décision purement politique qui autorisait la création d'une relation privilégiée entre le gouvernement et les communautés ethniques. La promotion des minorités ethniques par leur institutionnalisation au travers du multiculturalisme prouve que le Canada n'a pas échappé au piège du relativisme culturel pour enrayer la discrimination. Ainsi la notion de mosaïque, telle qu'elle est définie par la doctrine du bilinguisme et du multiculturalisme, porte les germes d'une menace supplémentaire d'éclatement de l'identité nationale. La vulnérabilité de l'identité canadienne, écartelée entre deux langues et deux cultures officielles et une société pluriculturelle, est mise au jour par la dissolution des valeurs communes au profit d'une tolérance souhaitable, mais qui ne peut à elle seule offrir la cohésion indispensable à l'unité de la collectivité (Bissonnette, 1991). Ici comme ailleurs, la discrimination positive doit être manipulée avec précaution afin d'éviter les détournements idéologiques possibles.

La logique du multiculturalisme, bien qu'animée d'un désir sincère d'équité sociale, se révèle génératrice de tension. Le principe de particularisme qui la sous-tend s'oppose profondément au principe d'universalisme sur lequel repose le système de valeurs et de traditions (valeurs liées à la citoyenneté, à la participation politique, à la justice, à l'égalité et aux droits humains en général) qui sert d'assise à la société libérale et démocratique canadienne (Hawkins, 1989). Cette reconnaissance politique entraîne la mutation des populations immigrées en minorités ethniques et comporte le risque d'un morcellement de la société en groupes ou coalitions d'intérêts, fondés sur l'ethnie ou la race, qui se disputent leur part des ressources limitées. Alors que la mission originale de la politique du multiculturalisme prétendait développer l'identité canadienne (les trois autres objectifs étaient la préservation des droits de la personne, le renforcement de la participation 
des citoyens, la fortification de l'unité canadienne tout en encourageant la diversification de la diversité culturelle dans un ensemble bilingue), I'accentuation de l'héritage culturel ne pouvait que saper à la base les valeurs identitaires canadiennes et compromettre l'engagement des nouveaux arrivants à l'égard de la société d'accueil.

L'année 1982 marque un nouveau départ pour la politique du multiculturalisme. Inspirée des politiques britanniques, dont la filiation est indéniable, la nouvelle orientation prétend enrayer ou atténuer le racisme. La politique de lutte contre la discrimination entérinée par les politiciens canadiens se révèle volontairement ambiguë, signifiant à la fois les éléments d'une politique d'assimilation capable de rétablir les droits individuels bafoués et un rejet de l'assimilation par une exaltation de la différence au nom du relativisme culturel. Une ethnicisation ou une racialisation des relations sociales et des cultures favorise le morcellement de la société canadienne.

À la diversité culturelle politiquement reconnue s'ajoute la brûlante question autochtone dont la résurgence au sein de l'actualité dévoile l'existence d'un racisme structurel proprement canadien que l'on tend trop souvent à oublier. Bien que ce problème diffère de celui que soulèvent les nouvelles immigrations, tous deux remettent en cause la représentation populaire de l'identité canadienne dans l'imaginaire collectif. De plus, les nouvelles immigrations dérangent, parce qu'elles modifient profondément le paysage humain des villes. Les conditions favorables à la fabrication de tensions sur fond racial sont réunies par le fait même (Jacquin, 1992: 165). Les difficultés d'intégration des nouveaux arrivants, même si elles découlent d'un manque de référence clair quant aux attentes de la société d'accueil (Bibeau, 1992), laissent de plus en plus percevoir l'immigration comme un difficile problème de gestion sociale et ne fait qu'amplifier la peur de l'Autre, vu comme inassimilable.

\section{INTERVENIR CONTRE LE RACISME}

La lutte contre le racisme en travail social est à la fois une exigence liée aux valeurs fondamentales du service social et aux missions qui lui sont confiées par l'État (Boucher, 1991a). Mais il ne suffit pas de mobiliser contre le racisme ou de créer des associations antiracistes pour y parvenir. Au contraire, comme nous venons de le voir, maintenant mouvements antiraciste et raciste se rejoignent par l'érection de barrières (culturelle ou raciale) entre les communautés. Sans analyse des enjeux politiques, des idéologies prônées par les uns comme par les autres et, surtout, des effets pervers de la défense des identités culturelles et du droit à la différence, l'intervenant risque, en toute bonne foi, de contribuer à ériger de nouvelles barrières et à attiser les tensions sociales et politiques entre des citoyens ethnicisés (Laperrière, 1990). 
Il se voit, de toute façon, de plus en plus souvent accuser de racisme par des clients qui affirment leurs différences au point de les ériger eux-mêmes en blocage, en barrière. La simple phrase: Vous ne pouvez pas comprendre, vous les blancs [en sous entendant vous les blancs, nationalistes québécois, de classe moyenne, ethnocentrique (Desrosiers, 1992)] est de plus en plus souvent entendu par les intervenants.

Système de blocage de la communication et de résistance aux changements dont la rentabilité politique pourrait être démontrée, l'affirmation de la différence et de l'incommunicabilité interculturelle se répand rapidement dans les services sociaux, soutenue par des perspectives théoriques supposément radicales et modernes.

Selon ces théoriciens, puisque le service social est essentiellement raciste (McMahon, 1992), n'est-on pas en droit de réclamer un service social culturalisé, ethnicisé, religieux (Boucher, 1988)? Puisque le Canada et le Québec sont multiculturels, il faut donner à chaque communauté culturelle les moyens, les institutions, les quartiers nécessaires pour «se protéger» de I'assimilation (Bibeau, 1992) à une supposée majorité oppressive, de plus en plus invisible.

En somme, selon ces auteurs, si les travailleurs sociaux n'orientent pas leur pratique vers la lutte contre l'oppression systémique des minorités par I'ensemble des institutions, ils ont une pratique raciste qui maintient la reproduction des conditions d'un racisme institutionnalisé (McMahon, 1992). $S^{\prime}$ il ne suffit pas d'adapter, d'intervenir en tenant compte de la différence culturelle, il faut, semble-t-il, que tous les établissements de service social et les intervenants se donnent pour mission de changer radicalement la société et s'impliquent politiquement en faveur des minorités (Coombe, 1986). Pour s'attaquer au racisme institutionnalisé, il suffit apparemment de commencer par modifier nos propres institutions (sous-entendu racistes), nos propres pratiques (sous-entendu racistes), et d'être en conformité avec la politique canadienne sur L'élimination de la discrimination raciale au Canada (1989) du Secrétaire d'État du Canada et du ministre d'État au multiculturalisme et à la citoyenneté.

Adhérer, sans discuter, à ce discours radical et fermé sur les pratiques antiracistes semble la seule façon acceptable de lutter. Pourtant, c'est un discours que nous qualifierons, avec Taguieff, de néo-raciste, surtout lorsqu'on I'applique uniformément à toutes les sociétés nord-américaines et européennes (Commission for racial equality, 1978). D'une part, se référer essentiellement au concept de race pour décrire des rapports sociaux, pour définir une société (Boucher, 1991b) est dépassé, malfaisant et dangereux (Somma, 1991 ; Paillé, 1992). D'autre part, la mobilisation politique (raciste ou antiraciste) sur une base ethnique, culturelle ou religieuse est I'une des formes les plus dangereuses du néo-racisme moderne et nous devons la dénoncer (Turenne, 
1993 ; Caccia, 1992). L'explication culturaliste des problèmes sociaux, des rapports sociaux est également suspecte (Labelle, 1990) et nous ne devons pas l'encourager.

Que pouvons-nous donc faire d'autre pour lutter efficacement contre le racisme sous toutes ses formes? Le risque d'une démobilisation des travailleurs sociaux face au racisme, mais aussi face aux mutations actuelles, est bien réel dans un contexte de crise intellectuelle, idéologique, organisationnelle, politique et économique (Wieviorka, 1993).

Heureusement, l'ethnicisation des postes de travail (Jacquin, 1992), des pratiques des établissements et de la citoyenneté est globalement refusée au Québec. Mais comment échapper à l'ethnicisation des relations sociales lorsque l'État y contribue en instituant les communautés culturelles comme «sujets ethnicisés» (Bibeau, 1992)?

Puisque nous avons tous des préjugés sur les «autres», ceux mis à distance par un nous/vous, transmis ou construit en généralisant une ou des expériences véçues, ces préjugés peuvent nous détourner de notre intention d'entrer en relation, de connaître et de comprendre «l'autre». Sous prétexte de respect de la différence, ces préjugés, combinés à des arguments culturalistes, serviront, si l'on n'y prend garde, à augmenter la distance, à ériger des barrières raciales infranchissables et alimenteront l'exclusion des clients sur une base culturelle, religieuse ou ethnique. Nous n'en sommes pas encore là heureusement (Sniderman, 1993). Mais déjà, faute de la reconnaissance de la fluidité des identités (Meintel, 1991) par les travailleurs sociaux, par les services publics et par l'État, nous contribuons à construire artificiellement des groupes ethniques qui risquent un jour de réclamer ici aussi (Les temps modernes, 1993) une nationalité ethnique.

Dans ce contexte, pour faire face au racisme populaire (Jacquin, 1992) et respecter la différence tout en évitant d'entrer dans des arguments culturalistes, nous proposons aux travailleurs sociaux, premièrement, d'éviter systématiquement d'utiliser le concept de race pour ne pas indirectement l'accréditer comme le font les antiracistes qui se définissent comme une « race» opprimée. «Le temps n'est plus aux programmes de sensibilisation. [...] Le racisme se combat sur le terrain, au niveau des comportements, des paroles et des gestes posés par chaque individu» (Latouche, 1991).

Deuxièmement, nous proposons d'utiliser la méthode d'analyse des différences sociales de Philippe Bernoux (1985: 180), qu'il résume ainsi :

Pour approcher les mentalités ou les cultures, il faut partir des systèmes d'action concrets. C'est sûrement la seule manière de comprendre les différences culturelles et internationales, mais c'est surtout la seule permettant $d^{\prime}$ obtenir un changement. ${ }^{1}$

1. C'est nous qui soulignons 
En effet, un changement est un phénomène difficile à réaliser, même s'il ne s'agit pas de changer l'autre (ce qui n'est pas éthiquement défendable), mais plutôt d'obtenir une amélioration de sa situation. En fait, dans toutes les cultures, tout changement est accepté uniquement dans la mesure où la personne pense qu'elle a des chances de gagner quelque chose ou, tout au moins qu'elle sent qu'elle maîtrise suffisamment les leviers et les conséquences du changement pour pouvoir y gagner quelque chose. Ceux qui ont le sentiment de perdre dans le changement ou d'y être forcé ne peuvent que le refuser ou tenter de le freiner. L'adaptation de l'intervention reste donc nécessaire pour pouvoir analyser et prendre en considération les différences personnelles et collectives concrètes (Boucher, 1993). La relation de confiance en contexte interculturel suppose la conscience réciproque de l'influence, sur soi, sur l'autre et sur la relation, des contextes économiques, sociaux, politiques et culturels différents.

Ensuite, nous pensons comme Michel Wieviorka (1993: 31) que:

Face à ces problèmes, les travailleurs sociaux peuvent contribuer à éviter la montée aux extrêmes, être localement des agents de citoyenneté, chercher à faire des identités spécifiques non pas des éléments de rejet de la modernité, mais au contraire autant de points d'appuis vers une modernité renouvelée.

Ils peuvent, en un mot, se dégager de leur crise intellectuelle à condition de se vouloir, quelles qu'en soient les difficultés, des opérateurs d'un aggiornamento du modèle français d'intégration, et non pas les spectateurs plus ou moins passifs de sa décomposition. Le problème n'est pas seulement qu'ils manquent de moyens, qu'ils relèvent $d^{\prime}$ institutions trop bureaucratiques, ou trop subordonnées au pouvoir politique, que leur formation est insuffisante. Il est avant tout dans leurs difficultés à reconnaître que les défis auxquels ils sont confrontés sont non plus seulement socio-économiques, comme par le passé, mais éclatés sur trois registres, social, certes, mais aussi politique et culturel, et qu'il leur faut, tout à la fois, agir sur ces trois fronts, et de manière à les articuler.

En somme, en prenant ses désirs pour des réalités, la pensée magique, combinée à I'angélisme (Bissonnette, 1992) ou aux extrémismes, ne peut qu'être une bonne alliée pour une propagation naïve du racisme au Québec. Les solutions aux problèmes des autochtones, des immigrants ou des groupes ethniques ne sont pas plus dans la multiplication des leaders politiques ethniques que dans la multiplication des services autochtones, immigrants ou ethniques (Latouche, 1993) ou dans I'assimilation imposée. C'est aux racines du racisme (Liauzu, 1992), au racisme de l'intelligence (Bourdieu, 1980) qu'il faudra d'abord s'attaquer.

Comment reconnaître le racisme sous toutes ses formes? Par la fonction invariante des racismes, la justification essentialiste, naturaliste des 
groupes du fait qu'ils d'existent comme ils existent, combinée à l'érection d'une barrière infranchissable entre soi et l'autre. On le reconnaît aussi par un recours obligé à des systèmes explicatifs abstraits comme barrière à la communication, à la relation ainsi que par l'abstraction, la généralisation combinée à l'absolutisation comme mécanisme de mise à distance.

Et comment combattre le racisme? En facilitant la multiplication des contacts interculturels dans un cadre sécurisant de communication interpersonnelle, en sortant des discours politiques fermés, en dénonçant l'ambiguïté conceptuelle volontairement entretenue par le ministère des Communautés culturelles et de I'Immigration ( $\mathrm{MCCl}$ ), le Secrétariat au multiculturalisme, certains leaders ethniques et certains chercheurs, en refusant de mesurer le racisme par les perceptions, les projections, le prêt d'intentions, en travaillant socialement pour la collectivité davantage que politiquement contre le racisme, le travailleur social ne risque pas trop de se tromper en produisant involontairement l'effet contraire à celui recherché.

L'intervention collective contre le racisme se construit sur le terrain, avec tous les citoyens concernés, là où les différences concrètes sont sources de tensions et d'insatisfactions, là où les systèmes de projection (Hall, 1976) s'affrontent, là où une autorité sécurisante fait défaut. (Doutreloux et Guilbert, 1992), là où... Le défi est alors de dépasser les confrontations d'évaluation réciproque pour parvenir à établir un esprit, un climat, un système de concertation autour d'objectifs communs. A priori, I'approche consensuelle doit être privilégiée. C'est possible et c'est efficace. C'est dans, par et pour l'action que les cultures sont créées (Bernoux, 1985); c'est donc aussi dans, par et pour l'action commune que les différences sont dépassées. Exceptionnellement, l'approche conflictuelle peut être préférable. L'intervention concerne alors un problème concret à résoudre et la mobilisation doit être la plus large possible, en accord avec des droits reconnus légalement pour tous les citoyens.

\section{CONCLUSION}

Le processus migratoire sud/nord semble inévitable du fait des déséquilibres économiques et démographiques structurels qui affligent le système international. Au cours des âges, différentes formes de domination (esclavagisme, colonialisme, impérialisme) ont permis aux pays industrialisés du Nord de recourir à des bassins de main-d'œuvre que leurs économies exigeaient. À partir de la crise des années 70, la diminution des facteurs d'attraction dans les pays d'accueil et l'augmentation des facteurs motivant le départ dans les pays du Sud ont contribué à une perte partielle de contrôle du processus migratoire. 
Les systèmes administratifs et juridiques nationaux et internationaux demeurent trop ancrés dans les concepts de territorialité, révélant une mésadaptation à la réalité d'une plus grande mobilité internationale stimulée par la mondialisation de l'économie et par l'adaptation de l'émigration aux nouveaux modèles de développement (Bolzmann, 1985: 68).

Les conséquences sur les migrants et par ricochet sur les pays d'accueil semblent elles aussi incontournables. Ces cultures dérangées par le processus migratoire, arrachées de leur élément naturel, deviennent, une fois transplantées, des cultures du dérangement dont la coexistence se révèle souvent complexe, mouvante, parfois brutale (Médam, 1989). Les sociétés nationales des pays d'immigration ont été modelées par les politiques propres à chaque État, mais celles-ci reflétaient également la structure inégalitaire du système international. Souvent le racisme structurel présent au sein d'une société n'est que la contrepartie «normale » $d^{\prime}$ un racisme politique qui a présidé à la hiérarchisation des nations sur le plan international. Actuellement, ces mêmes États accusent une certaine perte de contrôle tant sur le plan externe que sur le plan interne. Les nouvelles immigrations se révèlent alors problématiques, parce qu'elles s'incrustent dans des sociétés nationales déjà affaiblies par des mouvements de revendications identitaires ethniques qui effritent progressivement la cohésion sociale. La reconnaissance des différentes identités se fait paradoxalement au détriment d'une identité nationale qui est pourtant érigée en norme de référence.

Les nouvelles immigrations, par leur composition même, transcendent les frontières des États-nations. Les conflits sont de nationalité et de citoyenneté dans un contexte où la présence même de ces nouvelles populations installées dans les grandes métropoles attisent les débats en leur donnant de nouvelles dimensions. Avec René Gallisot (1989), nous sommes portées à croire que le multiculturel n'est qu'un simple feu follet courant sur une transformation de base: I'urbanisation généralisée et la généralisation des cultures urbaines avec comme corollaire le grossissement des relations interethniques. Ainsi s'opère un amalgame des cultures qui appelle une distinction de plus en plus marquée entre citoyenneté et nationalité, obligeant à penser une nouvelle citoyenneté plurielle:

Mais, n'est-ce pas que l'importance des diasporas, les recoupements des ensembles que sont ces nouveaux espaces d'échanges d'échelle continentale, méditerranéenne ou même transocéanique, qui se nouent ou se condensent dans la polarisation urbaine et dans la coexistence des signes identitaires, comme le procès de la transnationalisation culturelle poussent en avant une conception nouvelle de la citoyenneté? La citoyenneté échappe à la nationalité.

L'État-nation qui érige la nationalité, la sienne, en valeur universelle se trouve directement contesté par des mouvements minoritaires qui lui 
opposent l'absolutisation de leurs différences comme arme première. Entre ces deux extrêmismes, les principes républicains d'universalité disparaissent au profit d'une conception narcissique de l'identité collective. Le découplage citoyenneté/nationalité provoqué par les migrations internationales menace automatiquement l'intégrité de l'État-nation, tout comme la perte de souveraineté au profit des grands ensembles, des multinationales et des organisations financières internationales qui contrôlent les leviers économiques et la loi du marché sur le plan international.

La résurgence du racisme dans nos sociétés contemporaines pourrait très bien correspondre aux manifestations externes d'un difficile ajustement aux mutations sociales et politiques entraînées par l'arrivée de ces nouvelles immigrations. Alors, dans un contexte dominé de plus en plus par l'idéologie du relativisme culturel, comment penser la lutte contre le racisme?

Si le difficile cheminement vers une plus grande démocratisation se poursuit et que chacun, en tant que personne, mais aussi en tant que membre d'une collectivité donnée, participe à l'universalisation des droits de la personne par delà les différences de croyances, de couleurs de peau et de cultures, il importe de privilégier un type de communauté médiatrice d'universalité au détriment d'une communauté auto-suffisante, relevant d'un idéal protoraciste. Afin de réhabiliter l'idée républicaine dans les démocraties culturelles, il importe de désabsolutiser le principe de la différence, afin de l'intégrer comme facteur de relativisation dans un humanisme non ethnocentriste.

L'idée d'une communauté humaine est celle de la démocratie achevée, et non plus enclose dans les frontières d'un peuple-sujet, celles que requiert l'État-nation (Taguieff, 1987: 490).

Puisque le racisme peut être utilisé pour créer des tensions conduisant à briser la cohésion sociale et à casser la légitimité des États-nations, puisque, inversement, les tensions économiques et sociales sont des conditions favorisant la montée du racisme, c'est sans contredit l'un des problèmes que les travailleurs sociaux affronteront de plus en plus souvent en cette période de transition. Pour intervenir efficacement, ils ne pourront pas se contenter de soutenir (ou de détruire) les État-nations en référence à des modèles magiques d'intervention idéologique ou politique.

La montée récente du racisme n'est pas un effet du hasard. La culture ou la différence ne peut être appréhendée en dehors de son contexte historique, ni analysée sans tenir compte des agents qui la mettent en œuvre, à un moment historique donné. En sortant de l'abstraction et en comprenant davantage les processus concrets en cause, le rôle des acteurs en présence (Le Thank Koi, 1991), nous saurons mieux comment le combattre sans suivre naïvement les modèles culturalistes raciaux étrangers et sans accréditer des 
concepts ambigus. C'est par l'analyse et l'innovation que le service social deviendra capable de lutter efficacement contre le danger de la montée du racisme au Québec durant la période de transition et ensuite de contribuer à bâtir une société nouvelle plus égalitaire et plus juste.

\section{Références bibliographiques}

BEAUD, Stéphane et Gérard NOIRIEL (1991). "Penser "I'intégration" des immigrés », dans Face au racisme, Théories et hypothèses, tome I. Paris : Éditions La Découverte/Essais.

BERNOUX, Philippe (1985). La sociologie des organisations. Paris: Seuil.

BIBEAU, Gilles, A. M. CHAN-YIP, M. LOCK, C. ROUSSEAU et C. STERLIN, avec le concours de H. FleUry (1992). La santé mentale et ses visages, Un Québec pluriethnique au quotidien. Montréal: Gaétan Morin.

BISSONNETTE, Lise (1991). L'intégration des immigrants au Québec: entre la peur et l'angélisme. Allocution d'ouverture, Colloque des professeurs du $\mathrm{MCCl}$, Montréal, 19 septembre.

BISSONNETTE, Lise, "Le rapprochement ne suffit pas», Le Devoir, 8 avril 1992.

BolzmanN, C., A. JACQUES, G. JaCQUeS et A. MENETREy (1985). "Asile et immigration », dans La forteresse européenne et les réfugiés. Lausanne: Éditions d'en bas.

BOUCHER, Nicole (1988). "L'accessibilité des services aux communautés culturelles: principe ou réalité? », Service social, vol. 37, n 3.

BOUCHER, Nicole (1991a). "Les travailleurs sociaux sont-ils concernés par les politiques d'immigration et de multiculturalisme canadiennes? ", Le travailleur social, Ottawa, ACTS, vol. 58, n 4.

BOUCHER, Nicole (1991b). «Montréal, ville raciste?», Le Devoir, 19 août.

BOUCHER, Nicole (1993). "Société multiethnique: implication pour la déontologie et l'éthique professionnelle», Intervention, $\mathrm{n}^{\circ}$ 96, octobre.

BOURDIEU, Pierre (1980). "Le racisme de l'intelligence», dans Questions de sociologie. Paris : Éd. Minuit.

CACCIA, Fulvio (1992). «Canada: vers la balkanisation tranquille», Le monde diplomatique, octobre.

COMMISSION FOR RACIAL EQUALITY AND ASSOCIATION OF DIRECTORS OF SOCIAL SERVICES (1978). Multi-racial Britain : the Social Services Response : a Working Party Report. London: The Commission.

COOMBE, Vivienne et ALAN LITTLE (1986). Race and social work, a guide to training. London and New York: Tavistock Publications.

COSTA-LASCOUX, Jacqueline (1991). « Des lois contre le racisme», dans P.-A. Taguieff, Face au racisme, Théories et hypothèses, tome II. Paris: Éditions La Découverte/Essais. 
COSTA-LASCOUX, Jacqueline (1992). "Vers l'Europe des citoyens», dans Logiques d'État et immigrations. Paris: Éditions Kimé.

CROWLEY, John (1992). Logiques d'États et immigrations, "Consensus et conflits dans la politique de l'immigration et des relations raciales du Royaume-Uni ». Paris : Éditions Kimé.

DE PUDLER, Véronique (1991). "Seuil de tolérance et cohabitation pluriethnique», dans Face au racisme, Théories et hypothèses, tome II. Paris : Éditions La Découverte/Essais.

DESROSIERS, Éric (1992). "Nationalisme et racisme, Les nationalistes québécois se voient souvent accusés d'être racistes. L'accusation n'est pas fondée. », Le Devoir, 14 novembre.

DOUTRELOUX, Albert et Lucille GUILBERT (1992). "Communautés culturelles, une solidarité paradoxale », L'Action nationale, vol. LXXXII, $\mathrm{n}^{\circ} 8$.

GALLISOT, René (1989). «Au-delà du multiculturel : nationaux, étrangers et citoyens. Urbanisation généralisée et transnationalisation », Revue internationale $d^{\prime}$ action communautaire, 21/61.

HALL, Edward T. (1976). Au delà de la culture. Paris: Seuil.

HAWKINS, Fred (1989). Critical Years in Immigration, Canada and Australia Compared. Montréal, Kingston: McGill-Queen University Press.

JACQUIN, Daniel (1992). «Face au racisme populaire», Revue française des affaires sociales, $\mathrm{n}^{\circ} 46$, décembre.

LABELLE, Micheline (1990). «Pour résoudre le conflit amérindien, ne pas s'enfermer dans une vision raciale», Le Devoir, 14 septembre.

LAPERRIÈRE, Anne (1990). «Les leçons de la crise d'Oka. Sans courage ni imagination, le pays tourne de plus en plus au racisme», Le Devoir, 8 septembre.

LATOUCHE, Daniel (1989). "Le pluralisme ethnique et I'agenda public au Québec », Revue internationale d'action communautaire, 21/61.

LATOUCHE, Daniel (1991). «Une lutte qui doit commencer», Le Devoir, 3 août.

LATOUCHE, Daniel (1993). «Alice parmi nous», Le Devoir, 21 août.

LECA, Jean (1990). "Nationalités et citoyennetés dans l'Europe des immigrations", dans Logiques d'États et immigrations. Paris: Éditions Kimé.

LECA, Jean (1991). "La citoyenneté en question», Face au racisme, Théories et hypothèses, tome I. Paris : Éditions La Découverte/Essais.

Les temps modernes (1993). "La nationalité ethnique», août-septembre.

LE THANK Koi (1991). Culture, créativité et développement. Paris: L'Harmattan.

LIAUZU, Claude (1992). Race et civilisation. L'Autre dans la culture occidentale. Anthropologie critique. Paris: Syros.

MCMAHON, Anthony et Paula ALLEN-MEARES (1992). "Is social work racist? A content analysis of recent literature », Social work, vol. 37, n 6 .

MÉDAM, Alain (1989). "À propos du cosmopolitisme montréalais », Revue internationale d'action communautaire, 21/61.

MEINTEL, D. (1991). Identité et groupe ethnique: quelques réflexions. Montréal: manuscrit.

NOËL, Lise (1989). L'intolérance: une problématique générale. Montréal : Éditions Boréal. 
ORIOL, Michel (1989). «Modèles idéologiques et modèles culturels dans la reproduction des identités collectives en situation d'émigration », Revue internationale $d^{\prime}$ action communautaire, 21/61.

PAILLÉ, Yvon (1992). "Le concept de race: dépassé et malfaisant», Le Devoir, 11 novembre.

SECRÉTARIAT D'ÉTAT DU CANADA ET SECRÉTARIAT D'ÉTAT AU MULTICULTURALISME ET À LA CITOYENNETÉ (1989). L'élimination de la discrimination raciale au Canada. Ottawa.

SNiderman, Paul M., David A. NORTHRuP, Joseph F. FletCher, Peter H. Russell et Philip TETLOCK (1993). , «Psychological and cultural foundations of prejudice: The cas of anti-Semitism in Québec », Revue canadienne de sociologie et d'anthropologie, 30/2.

SOMMA, Hélène (1991). «Dans I'œil d'une immigrante. Non, les Québécois ne sont pas racistes", Le Devoir, 21 novembre.

TAGUIEFF, Pierre-André (1987). La force du préjugé. Essai sur le racisme et ses doubles. Paris : Éditions La Découverte/Essais.

TAGUIEFF, Pierre-André (1991). Face au racisme, Les moyens d'agir, tome I. Paris : Éditions La Découverte/Essais.

TAGUIEFF, Pierre-André (1991). « Les métamorphoses du racisme et la crise de l'antiracisme », dans Face au racisme, Théories et hypothèses, tome II. Paris : Éditions La Découverte/Essais.

TUGENDHAT, Ernst (1985). "La fin de la nuit», dans La forteresse européenne et les réfugiés. Éditions d'en bas: Lausanne.

TURENNE, Martine (1993). "Parizeau: en italien, en espagnol, ou en anglais armé de traducteurs, le chef péquiste rencontre la presse "ethnique" », Le Devoir, 25 août.

WEIL, Patrick (1992). «Convergences et divergences des flux», dans Logiques d'États et immigrations. Paris: Éditions Kimé.

WEIL, Patrick (1992). "Travail et droit d'asile», Le monde diplomatique, octobre.

VERHAEREN, Raphaël-Emmanuel (1990). Partir? Une théorie économique des migrations internationales. Grenoble, $316 \mathrm{p}$.

WiEviORKA, Michel (1991). "L'expansion du racisme populaire», dans Face au racisme, tome II. Paris : Éditions La Découverte/Essais.

WIEVIORKA, Michel (1993). "Universalisme et relativisme culturel, Crise et perspectives du travail social », dans AFSEA-93, Immigration et travail social, Journées d'étude de Paris. Paris: Association française pour la sauvegarde de l'enfance et de l'adolescence.

ZOLBERG, Aristide R. (1992). «Reforming the back door: perspectives historiques sur la réforme de la politique américaine d'immigration », dans Logiques d'États et immigrations. Paris: Éditions Kimé. 